\title{
Redes bayesianas aplicadas a las condiciones climáticas al interior de un invernadero con ventilación natural
}

\author{
Alejandra Álvarez-López ${ }^{1}$, Oscar Delfín-Santiesteban¹, Enrique Rico-García², \\ Guillermo De la Torre-Gea ${ }^{1}$ \\ ${ }^{1}$ CA Biosistemas, Universidad Tecnológica de Corregidora, \\ Querétaro, México \\ ${ }^{2}$ CA Ingeniería de Biosistemas, División Estudios de Posgrado, Facultad de Ingeniería, \\ Universidad Autónoma de Querétaro, \\ Santiago de Querétaro, Querétaro, México \\ gtorre@abanet.mx
}

\begin{abstract}
Resumen. La ventilación natural en invernaderos produce altas tasas de intercambio de aire; sin embargo, este comportamiento se producen cerca de las ventanas, lo que ocasiona un bajo intercambio de aire en la zona central del invernadero, debido a un efecto de estancamiento que reduce la distribución del viento en todo el invernadero. La predicción de los gradientes en un invernadero con ventilación natural es difícil de lograr, debido a la naturaleza inherentemente estocástica del flujo de aire. Las Redes bayesianas son técnicas numéricas de incertidumbre que se pueden utilizar para estudiar este problema. Se obtuvo un conjunto de datos experimentales: temperatura del aire, humedad del aire, velocidad del viento, y concentración de $\mathrm{CO} 2$ a uno y tres metros sobre el suelo, en el espacio de cultivo. El conjunto de datos fue discretizado y utilizado para desarrollar un modelo de Red Bayesiana que describe las relaciones entre las variables estudiadas. El modelo muestra las diferencias que nos permitan identificar el grado de dependencia de las variables, así como cuantificar su inferencia.
\end{abstract}

Palabras clave: Invernaderos, redes bayesianas, ventilación natural, clima.

\section{Introducción}

Es común que en los invernaderos se presenten temperaturas muy altas durante el verano. Este hecho provoca problemas para los agricultores que no tienen equipo de refrigeración para evitar el sobrecalentamiento. Al interior de los invernaderos, la distribución adecuada de los parámetros climáticos, tales como el flujo de aire, temperatura del aire, humedad del aire y la concentración de $\mathrm{CO}_{2}$, son los principales factores que influyen en la uniformidad de crecimiento de los cultivos. En la actualidad, se han realizado numerosos estudios sobre la ventilación natural, centrándose en la comprensión de las relaciones entre las variables que definen el clima dentro del invernadero, como se muestra a continuación. 
La velocidad del viento fuera del invernadero es un factor importante para la definición de flujo de aire y el clima dentro del invernadero. Las condiciones de frontera de la distribución de la velocidad del viento se deducen de los datos experimentales y de la dirección del viento con respecto al eje longitudinal del invernadero (De la torre- Gea et al., 2011b). Rico-García et al. (2006) mostraron que un invernadero con ventanas cenitales orientadas a la dirección del viento o barlovento funciona mejor que orientadas de forma opuesta o sotavento, mientras que en invernaderos con ventanas laterales sucede lo contrario, esto indica que la orientación del viento afecta el nivel de ventilación.

En un estudio realizado por Khaoua et al. (2006), pudieron determinar las tasas de ventilación entre 9 y 26,5 intercambios de aire por hora para el barlovento y 03.07 a 12.05 en la condición de sotavento, respectivamente. Un buen diseño puede mantener las condiciones climáticas aceptables y uniformes para los casos particulares en donde el viento es perpendicular al eje principal del invernadero. Por otra parte, la velocidad del viento presenta una influencia lineal con relación a las tasas de intercambio de aire, mientras que la dirección del viento no afecta en la misma magnitud. Majdoubi et al. (2009) determinaron que el flujo de aire por encima del cultivo es mayor que al interior y por debajo de éste. El viento al interior del invernadero propicia que el aire cálido y húmedo salga a través de las ventanas cenitales, sin embargo aún no se conoce cómo el aumento de la ventilación influye en una mejor uniformidad en las condiciones climáticas, debido a la escaza información sobre el movimiento del aire y su relación con la eficacia de la refrigeración y la uniformidad del medio ambiente (Sase, 2006).

El efecto de la radiación solar y temperatura a menudo se relacionan mediante el establecimiento de modelos que toman en cuenta el calentamiento de la pared y el calor específico del material con que está constituido el invernadero. La transferencia de radiación dentro del propio cultivo sigue siendo la principal preocupación, ya que determina las dos principales funciones fisiológicas de los cultivos: de transpiración y la fotosíntesis. Este problema actualmente no se ha descifrado y probablemente recibirá mucha atención en los próximos años (Bournet y Boulard, 2010). Pontikakos et al. (2006) analizaron los datos obtenidos a partir de un modelo de Dinámica de Fluidos Computacional (CFD), mostrando que la mayor temperatura externa es un parámetro fundamental que define el comportamiento en general de las temperaturas al interior del invernadero, mientras que la dirección del viento define las temperaturas en regiones específicas del invernadero.

Según Molina-Aiz et al. (2006), determinaron que la temperatura media del aire al interior del invernadero puede variar entre $28,2-32,9^{\circ} \mathrm{C}$, cuando una temperatura exterior es de $26^{\circ} \mathrm{C}$, presentándose variaciones de $13^{\circ} \mathrm{C}$ con respecto al exterior. Nebbali et al. (2006) utilizaron un método semianalítico para determinar el perfil de temperaturas del suelo a partir de los parámetros meteorológicos, para ayudar en la evaluación de cambio de flujo de calor entre la superficie del suelo y el aire. De acuerdo con los resultados de Majdoubi et al. (2009), la convección y la radiación son las formas dominantes de la transferencia de calor. Sus mediciones mostraron que la diferencia entre la temperatura del aire dentro y fuera del invernadero está fuertemente ligada a la radiación solar y en segundo lugar a la velocidad del viento.

Rico-García et al. (2008), mostraron que la ventilación en invernaderos debido al efecto de la temperatura produce altas tasas de intercambio de aire; Sin embargo, el 
movimiento del aire se producen cerca de las ventanas solamente, sin que haya intercambio de aire en la zona central de invernadero, debido a un efecto de estancamiento que reduce la distribución del viento en todo el invernadero. Por otra parte, Chow y Hold (2010) obtuvieron que la radiación térmica sin la participación del aire, altera la distribución de la temperatura del aire en la zona superior, y ésta a su vez afecta a la temperatura del aire por conducción y convección. Las condiciones térmicas de las paredes de un invernadero definen las altas temperaturas del aire, pero no afectan su distribución. La radiación juegan un papel importante en la distribución de calor y la humedad relativa influye en la transferencia de calor.

Algunos estudios como los de Roy y Boulard (2005), Roy et al. (2008), Campen (2008), Kim (2008), y Majdoubi et al. (2009) simularon las distribuciones de humedad dentro del invernadero, obteniendo buenas aproximaciones con diferentes métodos, que incluyen modelos de CFD. El estudio de humedad es importante para la interacción el cultivo y su entorno. Sólo unos pocos estudios han logrado obtener modelos de gradientes en invernaderos. Actualmente no existen modelos para predecir gradientes de $\mathrm{CO}_{2}$ siendo que influye directamente en la asimilación de los cultivos (Teittel et al., 2010).

La predicción de gradientes en un invernadero es difícil, debido a la naturaleza inherentemente estocástica del flujo de aire y por la cantidad de factores que influyen en la definición de las condiciones climáticas, por lo que es necesario incorporar nuevas técnicas que tomen en cuenta muchas variables a la vez. El objetivo de este estudio es abordar este problema mediante el enfoque de las Redes Bayesianas, para describir las relaciones entre las variables en un invernadero con ventilación deficiente. Las Redes bayesianas (BN por sus siglas en inglés) son técnicas numéricas de incertidumbre que hacen uso de la inferencia bayesiana como método heurístico (De la torre-Gea et al., 2011a).

\section{Redes bayesianas}

Las Redes Bayesianas (BN) son tipos de representación del conocimiento desarrollado en el campo de la inteligencia artificial para realizar aproximaciones en el campo del razonamiento (Pearl, 1988; Mediero, 2007; Gámez et al, 2011; Zaidan et $a l, 2011)$. Una BN es un gráfico acíclico cuyos nodos corresponden directamente a los conceptos o variables aleatorias y cuyos enlaces se corresponden con las relaciones o funciones (Correa et al., 2009). Las variables se definen en un dominio discreto o cualitativo, y las relaciones funcionales describen las inferencias causales expresadas en términos de probabilidades condicionales que se muestra en la ecuación (1):

$$
\mathrm{P}\left(\mathrm{x}_{1}, \ldots, \mathrm{x}_{\mathrm{n}}\right)=\prod_{i=1}^{n} \mathrm{P}\left(\mathrm{x}_{\mathrm{i}} \mid \text { parents }\left(\mathrm{x}_{\mathrm{i}}\right)\right)
$$

Una BN se puede utilizar para identificar las relaciones entre las variables anteriormente indeterminadas o para describir y cuantificar estas relaciones, incluso con un conjunto de datos incompletos (Hruschka et al, 2007; Reyes, 2010). El algoritmo de solución de BN permite el cálculo de la distribución de probabilidad esperada de las variables de salida. El resultado de este cálculo depende de las 
distribuciones de las probabilidades de las variables de entrada. A nivel global, las BN pueden ser percibidas como una distribución de probabilidad conjunta de una colección de variables aleatorias discretas (Garrote et al., 2007).

$$
\mathrm{P}\left(\mathrm{c}_{\mathrm{j}} \mid \mathrm{x}_{\mathrm{i}}\right)=\mathrm{P}\left(\mathrm{x}_{\mathrm{i}} \mid \mathrm{c}_{\mathrm{j}}\right) \mathrm{P}\left(\mathrm{c}_{\mathrm{j}}\right) / \sum_{k} \mathrm{P}\left(\mathrm{x}_{\mathrm{i}} \mid \mathrm{c}_{\mathrm{k}}\right) \mathrm{P}\left(\mathrm{c}_{\mathrm{k}}\right)
$$

Una probabilidad a priori $P\left(c_{j}\right)$ es una probabilidad de que una muestra xi pertenezca a la clase $c_{j}$, sin ninguna información sobre sus valores característicos, como se muestra en la ecuación (2). Las máquinas de aprendizaje en inteligencia artificial, están relacionadas con los métodos de minería de datos, la clasificación o agrupación y el reconocimiento de patrones. Los métodos estadísticos de aprendizaje automático se pueden aplicar al marco de la estadística bayesiana, sin embargo el aprendizaje automático se puede emplear en una variedad de técnicas de clasificación para producir otros modelos de $\mathrm{BN}$. El objetivo de una $\mathrm{BN}$ de aprendizaje es encontrar un arreglo de red que mejor describa los datos observados.

En los modelos de aprendizaje, el método más representativo es el de "búsqueda y resultado" basado en el algoritmo K2. Dicho algoritmo comienza asignado un nombre a cada variable sin "padres". A continuación agrega a cada variable un padre de forma incremental, la cual en su mayoría aumenta la puntuación de la estructura resultante. Cuando cualquier adición no puede aumentar el marcador, deja de aumentar padres a la variable. Tomando en cuenta un conocimiento previo del ordenamiento de las variables en base a su grado de dependencia, el espacio de búsqueda en esta restricción es mucho menor que el espacio de toda una estructura y no hay necesidad de comprobar los ciclos en el proceso de aprendizaje. Si no se tiene conocimiento anterior sobre el ordenamiento de las variables, se procede a nuevas búsquedas (Guoliang, 2009).

\section{Materiales y métodos}

Se realizaron muestreos y mediciones del flujo de aire mediante anemometría omnidireccional. Se obtuvo un conjunto de datos experimentales en un período de 36 horas comprendidos entre el 21 al 22 de agosto del 2011, mediante el empleo de sensores colocados en la parte central al interior del invernadero. El conjunto de datos se compone de las variables: Temperatura del aire, Humedad del aire, Velocidad del viento y Concentración de $\mathrm{CO}_{2}$. Las mediciones fueron obtenidas a dos alturas; a un metro en el interior del cultivo y a tres metros del suelo sobre el cultivo. Las mediciones de temperatura y humedad fueron realizadas a intervalos de cuatro minutos por medio de un sensor de tipo LM335. La concentración de $\mathrm{CO}_{2}$ fue determinada mediante un sensor de dióxido de carbono de tipo FYA600CO2H. La velocidad $\mathrm{y}$ dirección del aire fue determinada mediante anemómetros omnidireccionales, cuyo rango de operación es de 0 ms-1 a 20 ms- 1 con una precisión de $0,03 \mathrm{~m} \mathrm{~s}-1$. El conjunto de datos al interior del invernadero fueron tomados entre el 21 y 25 de agosto del 2011. Los datos fueron discretizados mediante el sistema ELVIRA, como se muestra posteriormente para ser empleados en el desarrollo del modelo de Redes Bayesianas que describe las relaciones entre todas las variables. 
El invernadero está localizado en la Universidad Autónoma de Querétaro, Campus Amazcala, la cual se ubica en las coordenadas siguientes: longitud $100^{\circ} 24^{\prime} \mathrm{W}$; latitud $20^{\circ} 36^{\prime} \mathrm{N}$; y altitud $1820 \mathrm{~m}$. La superficie del invernadero es de $964.8 \mathrm{~m}^{2}(26.8 \mathrm{~m}$ de ancho y $36 \mathrm{~m}$ de largo). El invernadero es de $5.49 \mathrm{~m}$ de altura y $4.2 \mathrm{~m}$ de altura a la canaleta, con orientación norte-sur. Cuenta con cuatro ventanas cenitales, uno en cada nave, $(0.9 \mathrm{~m}$ de ancho y $28 \mathrm{~m}$ de longitud) y cuatro ventanas de pared. Las ventanas en las paredes norte y sur son de $2.5 \mathrm{~m}$ de ancho y $20 \mathrm{~m}$ de longitud, y las ventanas de las paredes este y oeste son de $2.5 \mathrm{~m}$ de ancho y $28 \mathrm{~m}$ de longitud. Todas las ventanas son enrollables. Las ventanas cenitales y laterales constituyen el $10 \%$ y $24 \%$ de la cobertura total, respectivamente como se muestra en la Figura 1. Se empleó como cultivo la especie Lycopersicum pimpinellifolim, con una densidad de 2.7 plantas $/ \mathrm{m}^{2}$.

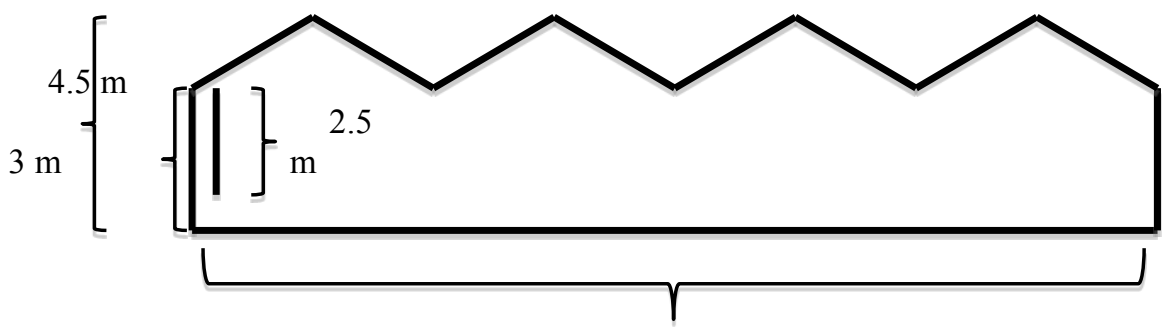

$26.8 \mathrm{~m}$

Fig. 1. Geometría del invernadero.

El comportamiento de las variables estudiadas fue similar tanto en los pasillos como al interior del cultivo, sin embargo, a un metro de altura, la temperatura fue más alta en el día y más baja en las noches, mientras que la humedad fue menor, como se muestra en la Fig. 2.

El análisis de BN fue realizado mediante el software ELVIRA versión 0.162 en tres etapas sugeridas por Mediero (2007).

1. Preprocesamiento: Se llevó a cabo mediante el algoritmo de imputación "por promedios" para completar las series de datos parciales. Este algoritmo reemplaza los valores faltantes o desconocidos, por el promedio de los valores para cada variable. Este método no necesita parámetros y consiste en la discretización de los datos masivos mediante el algoritmo, empleando dos intervalos con la misma frecuencia.

2. Procesamiento: De acuerdo con Wang et al. (2006), la mejor estructura de red bayesiana se obtiene empleando el algoritmo K2 con un número máximo de padres igual a 3 y sin restricciones.

3. Postprocesamiento: Se realizó un análisis de dependencias para obtener la estructura topológica de la red, la cual representa a las variables y sus dependencias causales. Después de obtener la red de aprendizaje paramétrico, se realizó el cálculo de las probabilidades condicionales en las variables que muestren relación o dependencia.

Posteriormente, el conjunto de datos fue analizado en intervalos de 3 horas, para desarrollar una BN de tiempo discreto. Para poder validar el modelo, se empleó un 
conjunto de datos diferentes al utilizado en la etapa de aprendizaje; las primeras mediciones fueron realizadas del 22 al 25 de agosto y correspondieron a las "Datos observados", el Segundo conjunto de datos fue medido entre el 18 y 21 de agosto y correspondieron a los "Datos esperados". El objetivo de esta prueba fue comprobar la $\mathrm{BN}$ para obtener una mejor solución, comparando las distribuciones de probabilidad.

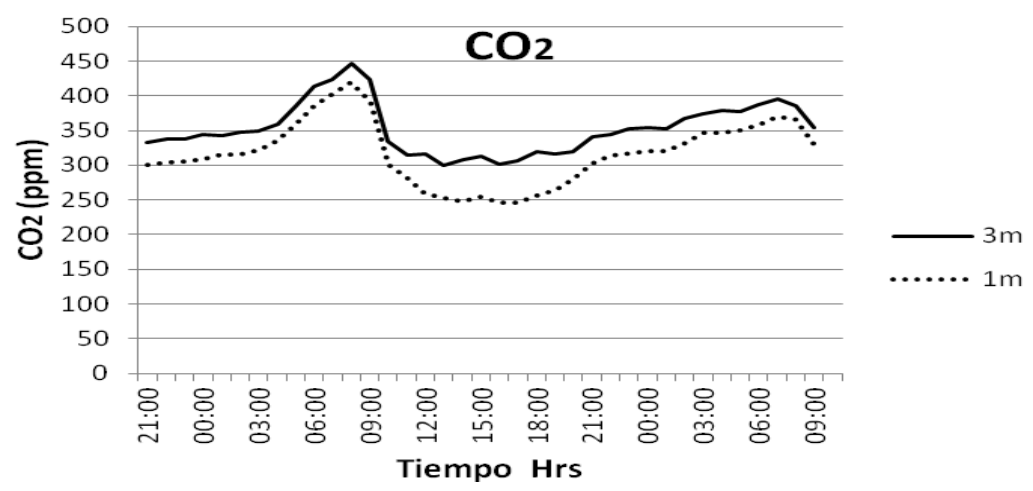

A) Concentración de $\mathrm{CO}_{2}(\mathrm{ppm})$.

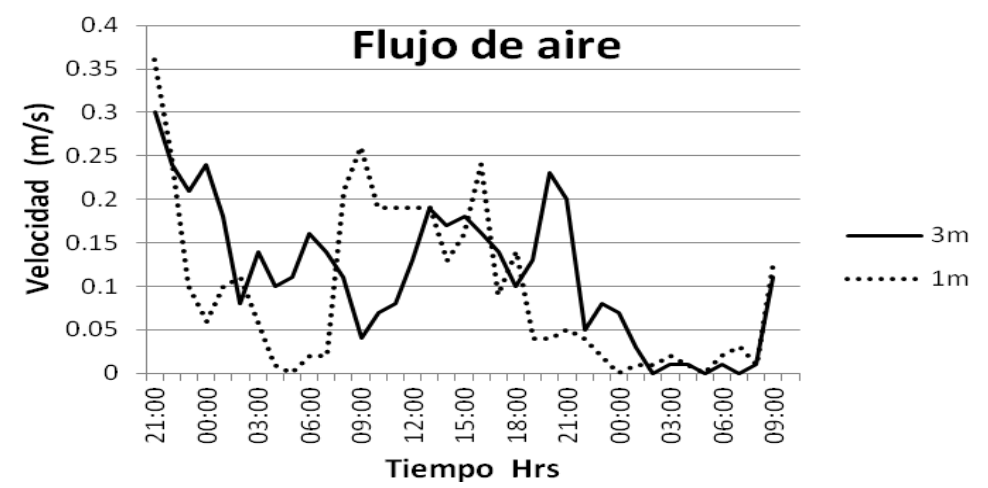

B) Velocidad del flujo de aire $(\mathrm{m} / \mathrm{s})$.

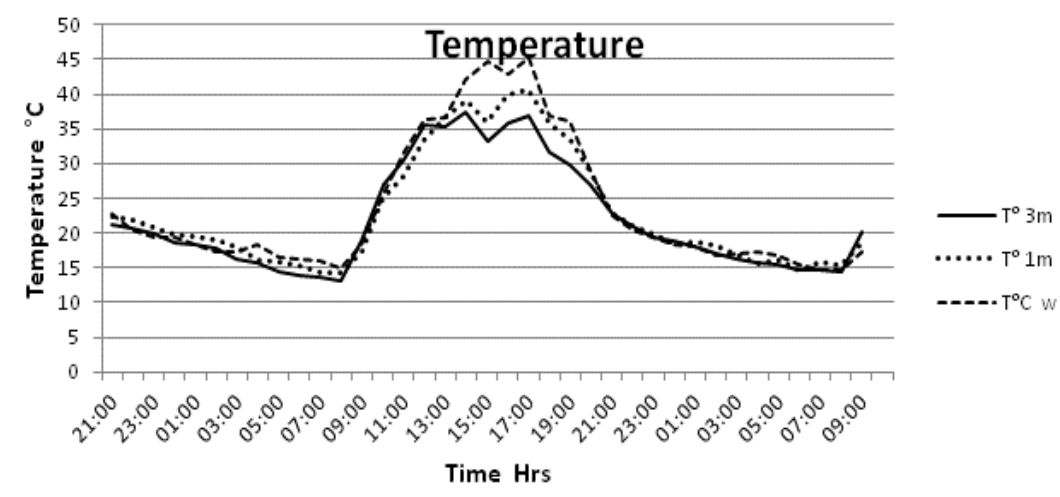

C) Temperatura media $\left({ }^{\circ} \mathrm{C}\right)$. 
Redes bayesianas aplicadas a las condiciones climáticas al interior de un invernadero ...

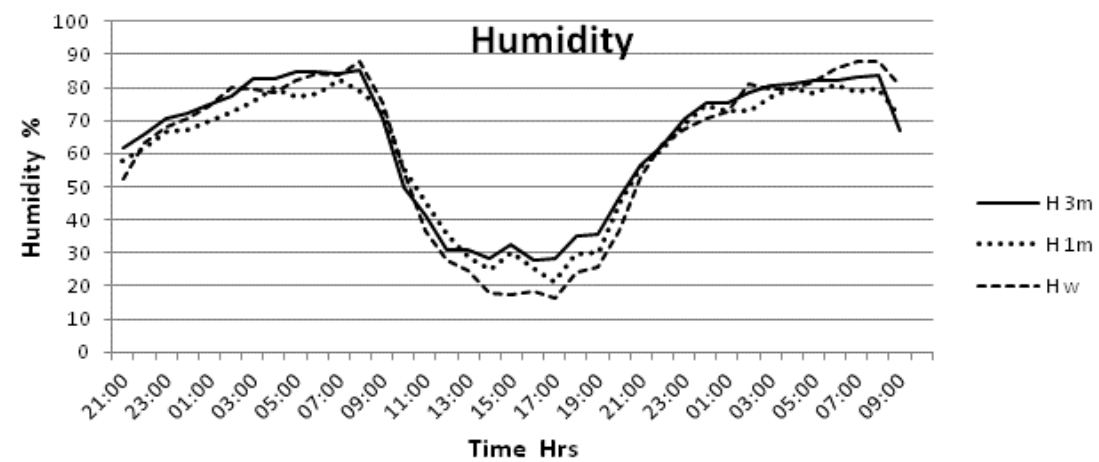

D) Humedad relativa (\%) a tres metros de altura ( $3 \mathrm{~m})$, un metro de altura (1m) y en los pasillos sin cultivo (w).

Fig. 2. Condiciones climáticas en la parte central del invernadero.

\section{Resultados y discusión}

Se obtuvo un modelo de BN con un $87.5 \%$ de precisión, calculado en la etapa de post-aprendizaje mediante el software ELVIRA, el cual muestra las relaciones entre las variables estudiadas. La Tabla 1. Describe los "Datos esperados" como los estados más probables de las variables. La validación del modelo de $\mathrm{BN}$ se muestra en la Tabla 2.

Tabla 1. Distribución de la probabilidad condicional entre $\mathrm{CO}_{2}$ sobre la temperatura del aire a 3 $\mathrm{m}$, temperatura del aire a $3 \mathrm{~m}$ sobre la humedad relativa a $1 \mathrm{~m}$, y $\mathrm{CO}_{2}$ a $3 \mathrm{~m}$ sobre $\mathrm{CO}_{2}$ a $1 \mathrm{~m}$.

\begin{tabular}{|c|c|c|c|c|c|c|c|c|c|c|c|}
\hline $\begin{array}{c}\mathrm{CO}_{2} \\
3 m \backslash \\
T^{o} 3 m\end{array}$ & $13^{\circ} \mathrm{C}$ & $25^{\circ} \mathrm{C}$ & $37^{\circ} \mathrm{C}$ & $\begin{array}{c}T^{o} \\
3 m \backslash \\
H 1 m\end{array}$ & $26 \%$ & $53 \%$ & $80 \%$ & $\begin{array}{c}\mathrm{CO}_{2} \\
3 \mathrm{~m} \backslash \mathrm{CO} \mathrm{O}_{2} \\
\mathrm{Im}\end{array}$ & $\begin{array}{c}245 \\
\text { ppm }\end{array}$ & $\begin{array}{c}323 \\
\text { ppm }\end{array}$ & $\begin{array}{c}400 \\
\text { ppm }\end{array}$ \\
\hline $\begin{array}{l}300 \\
\text { ppm }\end{array}$ & 0.067 & 0.133 & 0.75 & $13^{\circ} \mathrm{C}$ & 0.07 & 0.07 & 0.813 & $\begin{array}{l}300 \\
\text { ppm }\end{array}$ & 0.866 & 0.067 & 0.0625 \\
\hline $\begin{array}{c}375 \\
\text { ppm }\end{array}$ & 0.067 & 0.734 & 0.188 & $25^{\circ} \mathrm{C}$ & 0.07 & 0.8 & 0.125 & $\begin{array}{c}375 \\
\text { ppm }\end{array}$ & 0.067 & 0.866 & 0.0625 \\
\hline $\begin{array}{l}450 \\
\mathrm{ppm}\end{array}$ & 0.867 & 0.133 & 0.063 & $37^{\circ} \mathrm{C}$ & 0.87 & 0.13 & 0.063 & $\begin{array}{l}450 \\
\mathrm{ppm}\end{array}$ & 0.067 & 0.067 & 0.875 \\
\hline
\end{tabular}

Tabla 2. Validación del modelo de BN

\begin{tabular}{|c|c|c|}
\hline Relationship & Observed data & Expected data \\
\hline $\mathrm{CO}_{2} 3 \mathrm{~m} \backslash \mathrm{T}^{\mathrm{o}} \quad 3 \mathrm{~m}$ & $r=-0.999$ & $r=-0.999$ \\
\hline $\mathrm{T}^{\mathrm{o}} 3 \mathrm{~m} \backslash \mathrm{H} \quad 1 \mathrm{~m}$ & $r=-0.907$ & $r=-0.906$ \\
\hline $\mathrm{CO}_{2} 3 \mathrm{~m} \backslash \mathrm{CO}_{2} 1 \mathrm{~m}$ & $\mathrm{r}=0.999$ & $\mathrm{r}=0.986$ \\
\hline
\end{tabular}


Alejandra Álvarez-López, Oscar Delfín-Santiesteban, Enrique Rico-García, et al.
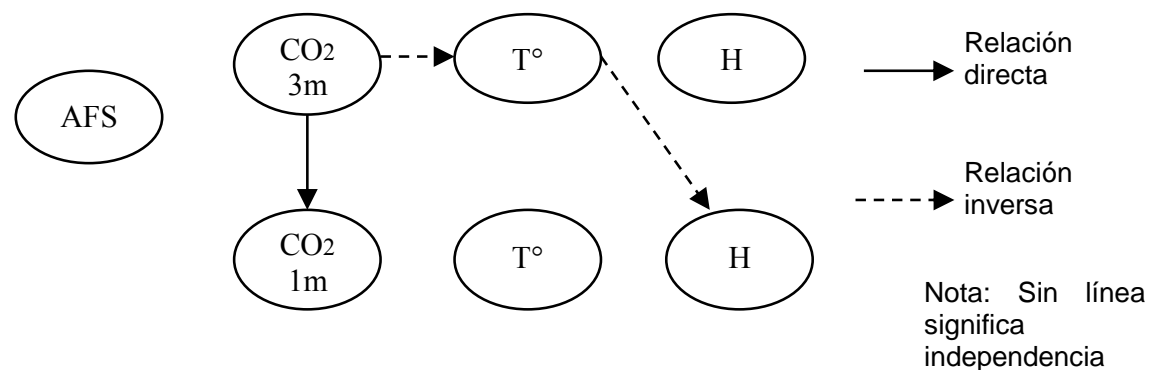

Fig. 3. Modelo de BN para un conjunto de datos de 36 horas en la parte central del invernadero: Velocidad del flujo de aire a $3 \mathrm{~m}$ (AFS $3 \mathrm{~m})$, concentración de $\mathrm{CO}_{2}$ a $1 \mathrm{~m}\left(\mathrm{CO}_{2} 1 \mathrm{~m}\right)$, concentración de $\mathrm{CO}_{2}$ a $3 \mathrm{~m}\left(\mathrm{CO}_{2} 3 \mathrm{~m}\right)$, Temperatura a $3 \mathrm{~m}\left(\mathrm{~T}^{\circ} \mathrm{C} 3 \mathrm{~m}\right)$, Temperatura a $1 \mathrm{~m}\left(\mathrm{~T}^{\circ} \mathrm{C}\right.$ $1 \mathrm{~m})$, Humedad relativa a $3 \mathrm{~m}(\mathrm{H} 3 \mathrm{~m})$, y Humedad relativa a $1 \mathrm{~m}(\mathrm{H} 1 \mathrm{~m})$.

En la Figura 3 se muestran las relaciones entre variables considerando tanto el día cómo la noche, por lo que es importante establecer éste modelo de forma parcial, ya que las relaciones entre variables no son las mismas en el día y en la noche, haciéndose necesario un análisis más detallado en intervalos de tiempo menores. Este modelo muestra que cuando la velocidad del aire es menor a $0.4 \mathrm{~m} / \mathrm{s}$ no afecta a otras variables. La concentración de $\mathrm{CO}_{2}$ a $1 \mathrm{~m}$ y $3 \mathrm{~m}$ están directamente relacionadas. La concentración de $\mathrm{CO}_{2}$ a $3 \mathrm{~m}$ y la Humedad Relativa a $1 \mathrm{~m}$ son inversamente proporcionales a la temperatura a $3 \mathrm{~m}$.

Este análisis mostró que la velocidad de flujo de aire no afecta las otras variables cuando la velocidad del aire es baja, ya que no promueve un intercambio de calor. Esto coincide con estudios previos de Rico-García et al. (2008), Majdoubi et al. (2009) y Chow y Hold (2010). Mientras que las concentraciones de $\mathrm{CO}_{2}$ situados a 1 $\mathrm{m}$ y $3 \mathrm{~m}$ son directamente proporcionales, el $\mathrm{CO}_{2}$ a $3 \mathrm{~m}$ es inversamente proporcional a la temperatura del aire a $3 \mathrm{~m}$, y del mismo modo la Humedad a $1 \mathrm{~m}$.

Las concentraciones de $\mathrm{CO}_{2}$ a 1 y 3 m están estrechamente relacionadas, tanto en la noche como en el día, el $\mathrm{CO}_{2}$ a $1 \mathrm{~m}$ funciona como una fuente de $\mathrm{CO}_{2}$ que incrementa su valor a $3 \mathrm{~m}$ elevándose rápidamente por encima del cultivo por diferencia de densidad. Durante el día, el cultivo consume $\mathrm{CO}_{2}$ inicialmente a 1 metro, y el incremento en la temperatura hace que el $\mathrm{CO}_{2}$ se vuelva menos denso y pasa a las capas superiores. No sucede lo mismo con la temperatura y la humedad, ya que tienen un comportamiento variable en el día y por la noche sobre 1 y $3 \mathrm{~m}$, con una temperatura más alta en el día y una menor humedad en $1 \mathrm{~m}$ que a $3 \mathrm{~m}$. Por la noche, estas relaciones se invierten, como se muestra en la Figura 4 (19:00 - 07:00). La relación inversamente proporcional del $\mathrm{CO}_{2}$ y la humedad con la temperatura a $3 \mathrm{~m}$ muestra que esta variable es la de mayor importancia, ya que su variación provoca cambios en otras variables tanto de día como de noche. Siendo la humedad a $1 \mathrm{~m}$ la variable más sensible.

07:00-10:00. La temperatura del aire a $3 \mathrm{~m}$ aumenta influyendo sobre la zona de cultivo, sobre la humedad del aire a 3 metros y la concentración de $\mathrm{CO}_{2}$ a $1 \mathrm{~m}$, las cuales disminuyen cuando la temperatura del aire aumenta y calienta las capas inferiores a $1 \mathrm{~m}\left(\mathrm{~T}^{\circ} \mathrm{C} \_\mathrm{BD}\right)$.

10:00-13:00. La concentración de $\mathrm{CO}_{2}$ a $1 \mathrm{~m}\left(\mathrm{CO}_{2}\right.$ B) disminuye debido al aumento en la temperatura del aire y la actividad fotosintética. La humedad del aire a 
$3 \mathrm{~m}$ muestra una relación directa con la humedad a $1 \mathrm{~m}$, que disminuye por el incremento de la temperatura. La concentración de $\mathrm{CO}_{2}$ a $3 \mathrm{~m}$ disminuye su valor, pero no bajo la influencia de la temperatura dentro del invernadero, posiblemente debido a la ventilación.

07:00 - 10:00

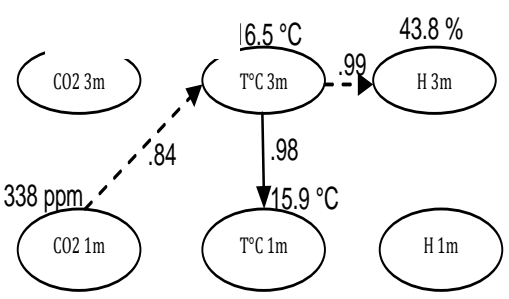

10:00-13:00

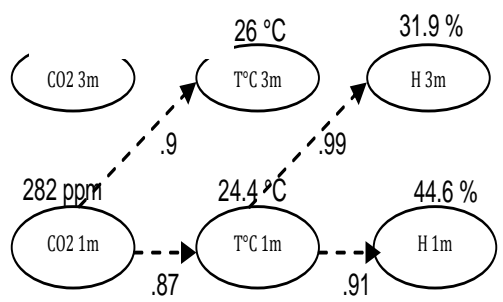

13:00-16:00

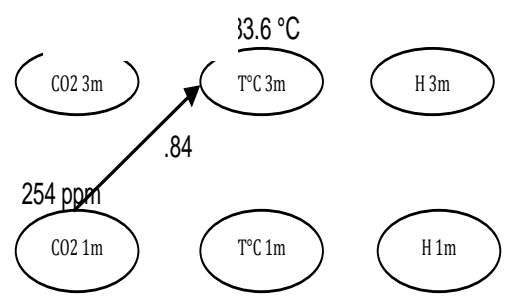

16:00-19:00

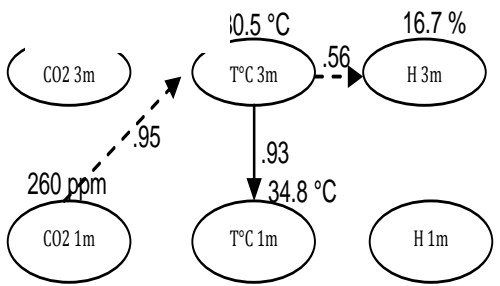

19:00 - 22:00

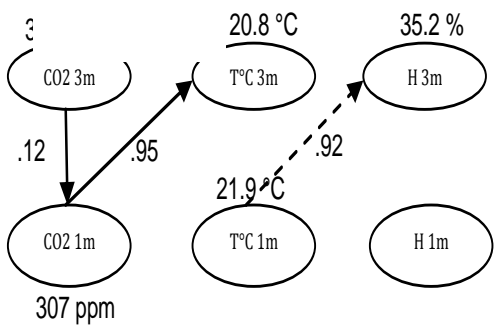

22:00-01:00

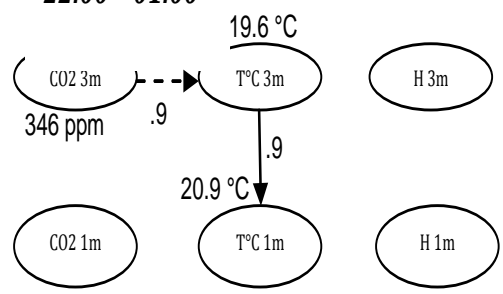

01:00 - 04:00

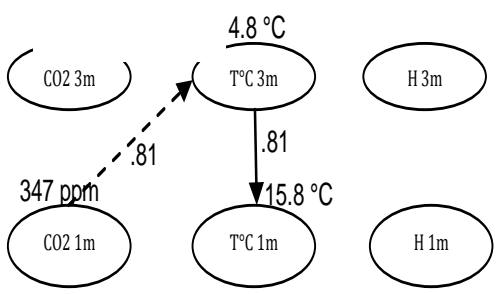

04:00-07:00

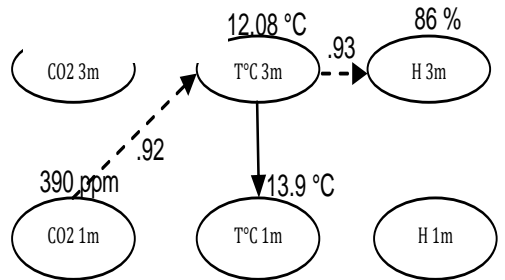

Fig. 4. Red Bayesiana para periodos de tres horas en un día. Concentración de $\mathrm{CO} 2$ a $3 \mathrm{~m}$ (CO2_A), concentración de $\mathrm{CO} 2$ a $1 \mathrm{~m}\left(\mathrm{CO} 2 \_\mathrm{B}\right)$, temperatura del aire a $3 \mathrm{~m}\left(\mathrm{~T}^{\circ} \mathrm{C} \_\mathrm{AD}\right)$, temperatura del aire a $1 \mathrm{~m}\left(\mathrm{~T}^{\circ} \mathrm{C} \_\mathrm{BD}\right)$, humedad relativa a $3 \mathrm{~m}\left(\mathrm{H} \_\mathrm{AD}\right)$, humedad a $1 \mathrm{~m}$ (H_BD). La línea continua indica una relación directa y la línea punteada indica una relación inversa. 
13:00-16:00. La temperatura máxima y valores mínimos de humedad del aire y el $\mathrm{CO}_{2}$ se alcanzó en el interior del invernadero en este período. La concentración de $\mathrm{CO}_{2}$ a $1 \mathrm{~m}(\mathrm{CO} 2$ B $)$ muestra influencia directa con la temperatura del aire de $3 \mathrm{~m}$ $\left(\mathrm{T}^{\circ} \mathrm{C} \_\mathrm{AD}\right)$ lo cual es indicativo de la suspensión en la fotosíntesis por sobre calentamiento.

16:00-19:00. Baja la temperatura del aire y la fotosíntesis de la plantas se expresa una vez más en la relación inversa entre el $\mathrm{CO}_{2}$ a 1 metro $\left(\mathrm{CO} 2 \_\mathrm{B}\right)$ y la temperatura del aire de $3 \mathrm{~m}\left(\mathrm{~T}^{\circ} \mathrm{C}_{-} \mathrm{AD}\right)$. De forma inversa al período entre las $\overline{7}: 00$ a 10:00, cuando la temperatura del aire a $3 \mathrm{~m}$ disminuye, la temperatura del aire a $1 \mathrm{~m}\left(\mathrm{~T} \mathrm{C}_{-} \mathrm{BD}^{\circ}\right)$ también reduce sus valores desde este punto hasta el amanecer del día siguiente. La humedad del aire comienza a incrementarse.

19:00-22:00. El sol se oculta y las plantas paran la fotosíntesis, lo cual se expresa mediante la relación inversa entre la temperatura del aire a $3 \mathrm{~m}\left(\mathrm{~T}^{\circ} \mathrm{C} \_\mathrm{AD}\right)$, y el $\mathrm{CO}_{2}$ a $1 \mathrm{~m}(\mathrm{CO} 2$ B $)$ el cual se incrementa por la respiración de las plantas, aumenta la concentración de $\mathrm{CO} 2$ a $3 \mathrm{~m}(\mathrm{CO} 2-\mathrm{A})$. La temperatura del aire a $1 \mathrm{~m}\left(\mathrm{~T}^{\circ} \mathrm{C} \_\mathrm{B}\right)$ es mayor que a $3 \mathrm{~m}\left(\mathrm{~T}^{\circ} \mathrm{C} \_\mathrm{A}\right)$, y por lo tanto disminuye, presentando relación inversa con la humedad del aire a $3 \mathrm{~m}$ (H_AD), la cual aumenta.

22:00-01:00. La tendencia es similar al período anterior, sin embargo la concentración de $\mathrm{CO} 2$ en $1 \mathrm{~m}$ muestra la relación inversa con la temperatura del aire en $3 \mathrm{~m}$ debido a la respiración.

01:00-04:00. El $\mathrm{CO}_{2}$ a $1 \mathrm{~m}$ muestra la relación inversa con la temperatura del aire en $3 \mathrm{~m}$ debido a la respiración.

04:00-07:00. En este punto, se han logrado mayores niveles de concentración de $\mathrm{CO}_{2}$, la humedad del aire y disminuye la temperatura del aire. Al final de este período se tiene la mayor diferencia en la humedad del aire a los $3 \mathrm{~m}$ y a $1 \mathrm{~m}$. Se registra la temperatura más baja a $3 \mathrm{~m}$, mostrando relación inversa con el mayor nivel de humedad a $1 \mathrm{~m}$, lo que sugiere que la humedad sube por efecto de la respiración del cultivo.

\section{Conclusión}

Utilizando un modelo de BN es posible observar y cuantificar las relaciones entre las variables Temperatura, Humedad relativa, velocidad del flujo de aire y la concentración de $\mathrm{CO}_{2}$. Modelo de $\mathrm{BN}$ muestra que la Velocidad de flujo de aire no afecta a las otras variables cuando la velocidad del aire es baja. Los modelos de BN en tiempo discreto muestran las relaciones entre las variables que sugieren los procesos fisiológicos del cultivo y la interacción con su entorno. El estado de un proceso fisiológico en el cultivo está representado por un valor de probabilidad condicional en un intervalo de tiempo determinado y éste cambia a lo largo del día. Mediante un modelo de BN es posible conceptualizar el espacio de cultivo como un subsistema diferente a los pasillos y en el área por encima del cultivo, que interactúan con su ambiente dentro del invernadero. Las distribuciones de probabilidad condicionales son una medida cuantitativa de las relaciones entre las variables y muestran el estado más probable de estas variables. Los modelos de $\mathrm{BN}$ tienen la 
capacidad de mostrar las relaciones entre las variables que involucran procesos fisiológicos de los cultivos en el interior de un invernadero.

Agradecimientos. El último autor es el autor para correspondencia. Este trabajo fue parcialmente financiado por el Consejo Nacional de Ciencia y Tecnología (CONACyT), la Universidad Autónoma de Querétaro y la Universidad Tecnológica de Corregidora.

\section{Referencias}

1. Bournet, P.E., Boulard, T.: Effect of ventilator configuration on the distributed climate of greenhouses: A review of experimental and CFD studies. Comput Electron Agric, 74, 195 (2010)

2. Campen, J. B.: Vapor removal from the greenhouse using forced ventilation when applying a thermal screen. Acta Hortic, 801, 863 (2008)

3. Correa, M., Bielza, C., Paimes-Teixeira, J., Alique, J. R.: Comparison of Bayesian networks and artificial neural networks for quality detection in a machining process. Expert Syst Appl, 36(3), 7270 (2009)

4. Chow, K., Hold, A.E.: On the influence of boundary conditions and thermal radiation on predictive accuracy in numerical simulations of indoor ventilation. Building and Environment, 45, 437 (2010)

5. De la Torre-Gea, G., Soto-Zarazúa, G.M., Guevara-González, R., Rico-García, E.: Bayesian Networks for defining relations-hips among climate factors. IJPS, 6(18) 4412 (2011)

6. De la Torre-Gea, G., Soto-Zarazúa, G.M., López-Crúz, I., Torres-Pacheco, I., Rico-García, E.: Computational fluid dynamics in greenhouses: A review. AJB, 10(77), 17651 (2011)

7. Gámez, J.A., Mateo, J.L., Puerta, J.M.: Learning Bayesian networks by hill climbing: efficient methods based on progressive restriction of the neighborhood. Data Min. Knowl. Discov, 22, 106 (2011)

8. Garrote, L., Molina, M., Mediero, L.: Probabilistic Forecasts Using Bayesian Networks Calibrated with Deterministic Rainfall-Runoff Models. In: Vasiliev et al., Extreme Hydrological Events: New Concepts for Security. Springer, 173 (2007)

9. Guoliang, L.: Knowledge Discovery with Bayesian Networks, Ph. D. thesis, National University of Singapore, Singapore (2009)

10. Hruschka, E., Ebecken, N.F.F.: Bayesian networks for imputation in classification Problems. J Intell Inform Syst, 29, 231 (2007)

11. Khaoua, S.A.O., Bournet, P.E., Migeon, C., Boulard, T., Chassériaux, G.: Analysis of greenhouse ventilation efficiency based on computational fluid dynamics. Biosystems Eng, 95, 83 (2006)

12. Kim, K., Yoona, J.Y., Kwonb, H.J., Hanna, J.H., Sonc, J.E., Namd, S.W., Giacomelli, G.A., Lee, I.B.: 3-D CFD analysis of relative humidity distribution in greenhouse with a fog cooling system and refrigerative dehumidifiers. Biosystems Eng, 100, 245 (2008)

13. Majdoubi, H., Boulard, T., Fatnassi, H., Bouirden, L.: Airflow and microclimate patterns in a one-hectare canary type greenhouse: an experimental and CFD assisted study, Agr. Forest Meteorol, 149, 1050 (2009) 